\title{
Survey on the $p$-adic Hayman conjecture
}

\author{
by Alain Escassut and Jacqueline Ojeda
}

\section{Introduction}

We denote by $\mathbb{K}$ an algebraically closed field of characteristic 0 , complete with respect to an ultrametric absolute value $\mid$. | and by $\mathbb{K}(x)$ the field of rational functions with coefficients in $\mathbb{K}$. Given $a \in \mathbb{K}$ and $R \in \mathbb{R}_{+}^{*}$, we denote by $d(a, R)$ the disk $\{x \in$ $\mathbb{K}|| x-a \mid \leq R\}$, by $d\left(a, R^{-}\right)$the disk $\{x \in \mathbb{K}|| x-a \mid<R\}$ and by $C(a, r)$ the circle $\{x \in \mathbb{K}|| x-a \mid=r\}$. Next, we denote by $\mathcal{A}(\mathbb{K})$ the $\mathbb{K}$-algebra of analytic functions in $\mathbb{K}$ (i.e. the set of power series with an infinite radius of convergence) and by $\mathcal{M}(\mathbb{K})$ the field of meromorphic functions in $\mathbb{K}$ (i.e. the field of fractions of $\mathcal{A}(\mathbb{K})$ ). Similarly, we denote by $\mathcal{A}\left(d\left(a, R^{-}\right)\right)$the $\mathbb{K}$-algebra of analytic functions in $d\left(a, R^{-}\right)$(i.e. the set of power series with a radius of convergence $\geq R)[8],[11],[12],[13]$ and by $\mathcal{M}\left(d\left(a, R^{-}\right)\right)$ its field of fractions and we denote by $\mathcal{A}_{b}\left(d\left(a, R^{-}\right)\right)$the $\mathbb{K}$-algebra of bounded analytic functions in $d\left(a, R^{-}\right)$and by $\mathcal{M}_{b}\left(d\left(a, R^{-}\right)\right)$its field of fractions and we set $\mathcal{A}_{u}\left(d\left(a, R^{-}\right)\right)=$ $\mathcal{A}\left(d\left(a, R^{-}\right)\right) \backslash \mathcal{A}_{b}\left(d\left(a, R^{-}\right)\right)$and $\mathcal{M}_{u}\left(d\left(a, R^{-}\right)\right)=\mathcal{M}\left(d\left(a, R^{-}\right)\right) \backslash \mathcal{M}_{b}\left(d\left(a, R^{-}\right)\right)$.

Given $f \in \mathcal{M}(\mathbb{K})$ (resp. $f \in \mathcal{M}_{u}\left(d\left(a, R^{-}\right)\right)$), a value $b \in \mathbb{K}$ is called an exceptional value for $f$ if $f-b$ has no zero in $\mathbb{K}$ (resp. in $d\left(a, R^{-}\right)$) and it is called a quasi-exceptional value for $f$ if $f-b$ has finitely many zeros in $\mathbb{K}$ (resp. in $d\left(a, R^{-}\right)$).

In the complex field, in the fifties, Walter Hayman asked the question whether, given a meromorphic function $g$ in the whole plane $\mathbb{C}$ and an integer $n \in \mathbb{N}$, the function $g^{\prime} g^{n}$ might admit an exceptional value $b \neq 0$ or a quasi-exceptional value $b \neq 0$ [10]. W. Hayman showed that $g^{\prime} g^{n}$ has no quasi-exceptional value, whenever $n \geq 3$. Next, the problem was solved for $n=2$ by E. Mues in 1979 [15] and next, for $n=1$, in 1995 by W. Bergweiler and A. Eremenko [1] and separately by H. Chen and M. Fang.

The same problem occurs on the field $\mathbb{K}$, both in $\mathcal{M}(\mathbb{K})$ and in a field $\mathcal{M}\left(d\left(a, R^{-}\right)\right), a \in$ $\mathbb{K}, R>0$. Several basic results will be necessary to examine this.

In ultrametric analysis as in complex analysis, we have this immediate correspondance:

Lemma 1: Let $g \in \mathcal{M}(\mathbb{K})$ (resp. let $g \in \mathcal{M}\left(d\left(a, R^{-}\right)\right.$), $\left.a \in \mathbb{K}, R>0\right)$, let $f=\frac{1}{g}$ and let $n \in \mathbb{N}^{*}$. Then $g^{\prime} g^{n}$ admits a quasi-exceptional value $b \in \mathbb{K}^{*}$ if and only if $f^{\prime}+b f^{n+2}$ has finitely many zeros that are not zeros of $f$.

Remark: We can also consider the same problem when $n=-1$ i.e. the question whether $f^{\prime}+b f$ has infinitely many zeros. In $\mathbb{C}$ the well known counter-example furnished by the function $\exp (x)$ shows that $f^{\prime}+f$ may have no zero. When $n=0$, in $\mathbb{C}$ the well known counter-example furnished by the function $\tan (-x)$ shows that $f^{\prime}+f^{2}$ may have no zero. On the field $\mathbb{K}$, we will examine the cases $n=-1$ and $n=0$. 
Henceforth, for $n \geq 3$, we will examine that problem by considering the set of zeros of $f^{\prime}+b f^{n+2}$, with $b \neq 0$. In the field $\mathbb{K}$, two theorems are specific to p-adic analysis. Both are based on the following lemma. We set $m=n+2$.

Notation: Let $f \in \mathcal{M}\left(d\left(0, R^{-}\right)\right)$. For every $\left.r \in\right] 0, R[,|f(x)|$ has a limit when $|x|$ tends to $r$ while staing different from $r$ and that limit is denoted by $|f|(r)$ [8].

Let $\mu=\log r$. We set $\Psi(f, \mu)=\log (|f|(r))$ We denote by $\nu^{+}(f, \mu)$ the difference between the number of zeros and the number of poles of $f$ in $d(0, r)$ and we denote by $\nu^{-}(f, \mu)$ the difference between the number of zeros and the number of poles of $f$ in $d\left(0, r^{-}\right)$.

The following Lemma 1 and 2 are classical [17]:

Lemma 2: Let $f \in \mathcal{M}(\mathbb{K})$. Then $\nu^{+}(f, \mu)$ is the right side derivative of $\Psi(f, \mu)$ and $\nu^{+}(f, \mu)$ is the left side derivative of $\Psi(f, \mu)$.

Lemma 3: Let $f \in \mathcal{M}(\mathbb{K})$, (resp. let $f \in \mathcal{M}\left(d\left(a, R^{-}\right)\right)$, $\left.a \in \mathbb{K}, R>0\right)$, suppose that $f$ admits infinitely many zeros and suppose that there exists a sequence of intervals $\left[r_{n}^{\prime}, r_{n}^{\prime \prime}\right]$ such that $\lim _{n \rightarrow+\infty} r_{n}^{\prime}=+\infty$ (resp. $\lim _{n \rightarrow+\infty} r_{n}^{\prime}=\lim _{n \rightarrow+\infty} r_{n}^{\prime \prime}=R$ ) and such that $\left|\left(f^{\prime}+f^{m}\right)\right|(r)=$ $\left|f^{m}\right|(r) \forall r \in \bigcup_{n \in \mathbb{N}}\left[r_{n}^{\prime}, r_{n}^{\prime \prime}\right]$. Let $m \in \mathbb{N}^{*}$ be $\neq 2$. Then $f^{\prime}+f^{m}$ has infinitely many zeros that are not zeros of $f$.

Proof: Let $J=\bigcup_{n \in \mathbb{N}}\left[r_{n}^{\prime}, r_{n}^{\prime \prime}\right]$. When $r$ is big enough, we have $\left|f^{m}\right|(r)>\left|f^{\prime}\right|(r)$ therefore

$$
\nu^{+}\left(f^{\prime}+f^{m}, \log r\right)=\nu^{+}\left(f^{m}, \log r\right), \nu^{-}\left(f^{\prime}+f^{m}, \log r\right)=\nu^{-}\left(f^{m}, \log r\right) \forall r \in J
$$

Consequently, in each disk $d(0, r)$ with $r \in J, f$ and $f^{\prime}+f^{m}$ have the same difference between the number of zeros and poles. Now, if $m \geq 3$ the poles of $f^{\prime}+f^{m}$ and $f^{m}$ are the same taking multiplicity into account. And when $m=1$, each pole of $f$ is a pole of $f^{\prime}+f$ with a greater order. Consequently, for each $r \in J$, the number of zeros of $f^{\prime}+f^{m}$ in $d(0, r)$ is superior or equal to this of $f^{m}$.

Now, for each $n \in \mathbb{N}$, let $s_{n}$ be the number of distinct zeros of $f$ in $d\left(0, r_{n}^{\prime \prime}\right)$. Since $f$ has infinitely many zeros, the sequence $s_{n}$ is increasing and tends to $+\infty$. On the other hand, for each zero $\alpha$ of order $u$ of $f$, either $\alpha$ is not a zero of $f^{\prime}+f^{m}$ (when $u=1$ ), or it is a zero of order $u-1$. Consequently, the number of zeros of $f^{\prime}+f^{m}$ in $d\left(0, r_{n}^{\prime \prime}\right)$ which are not zeros of $f$ is at least $s_{n}$. Thus we have proved that $f^{\prime}+f^{m}$ has infinitely many zeros that are not zeros of $f$.

Remark: Relation (1) above does not hold when $m=2$ because poles of $f^{2}$ and $f^{\prime}$ may have the same order and therefore may kill each other.

In most of results, we will use the ultrametric Nevanlinna theory [33], [35]. The Nevanlinna Theory was made by Rolf Nevanlinna on complex functions [16], [10]. It 
consists of defining counting functions of zeros and poles of a meromorphic function $f$ and giving an upper bound for multiple zeros and poles of various functions $f-b, b \in \mathbb{C}$.

A similar theory for functions in a p-adic field was constructed by A. Boutabaa [6].

Notations: Given three functions $\phi, \psi, \zeta$ defined in an interval $J=] a,+\infty[$ (resp. $J=] a, R[)$, with values in $[0,+\infty[$, we shall write $\phi(r) \leq \psi(r)+O(\zeta(r))$ if there exists a constant $b \in \mathbb{R}$ such that $\phi(r) \leq \psi(r)+b \zeta(r)$. We shall write $\phi(r)=\psi(r)+O(\zeta(r))$ if $|\psi(r)-\phi(r)|$ is bounded by a function of the form $b \zeta(r)$.

Similarly, we shall write $\phi(r) \leq \psi(r)+o(\zeta(r))$ if there exists a function $h$ from $J=$ ]$a,+\infty[$ (resp. from $J=] a, R\left[\right.$ ) to $\mathbb{R}$ such that $\lim _{r \rightarrow+\infty} \frac{h(r)}{\zeta(r)}=0\left(\operatorname{resp} . \lim _{r \rightarrow R} \frac{h(r)}{\zeta(r)}=0\right)$ and such that $\phi(r) \leq \psi(r)+h(r)$. And we shall write $\phi(r)=\psi(r)+o(\zeta(r))$ if there exists a function $h$ from $J=] a,+\infty[$ (resp. from $J=] a, R\left[\right.$ ) to $\mathbb{R}$ such that $\lim _{r \rightarrow+\infty} \frac{h(r)}{\zeta(r)}=0$ (resp. $\left.\lim _{r \rightarrow R} \frac{h(r)}{\zeta(r)}=0\right)$ and such that $\phi(r)=\psi(r)+h(r)$.

The p-adic Nevanlinna Theory was first stated and correctly proved by A. Boutabaa in $\mathcal{M}(\mathbb{K})$ [6]. In [7] the theory was extended to functions in $\mathcal{M}\left(d\left(0, R^{-}\right)\right)$by taking into account Lazard's problem [14].

Throughout the next paragraphs, we will denote by $I$ the interval $[t,+\infty[$ and by $J$ an interval of the form $[t, R[$ with $t>0$.

We have to introduce the counting function of zeros and poles of $f$, counting or not multiplicity. Here we will choose a presentation that avoids assuming that all functions we consider admit no zero and no pole at the origin.

Definitions: We denote by $Z(r, f)$ the counting function of zeros of $f$ in $d(0, r)$ in the following way:

Let $\sigma(r)$ be the number of distinct zeros of $f$ in $d(0, r)$ and let Let $\left(a_{n}\right), 1 \leq n \leq \sigma(r)$ be the finite sequence of zeros of $f$ in $d(0, r)$, of respective order $s_{n}$.

We set $Z(r, f)=\max \left(\omega_{0}(f), 0\right) \log r+\sum_{n=1}^{\sigma(r)} s_{n}\left(\log r-\log \left|a_{n}\right|\right)$ and so, $Z(r, f)$ is called the counting function of zeros of $f$ in $d(0, r)$, counting multiplicity.

In order to define the counting function of zeros of $f$ without multiplicity, we put $\overline{\omega_{0}}(f)=0$ if $\omega_{0}(f) \leq 0$ and $\overline{\omega_{0}}(f)=1$ if $\omega_{0}(f) \geq 1$.

Now, we denote by $\bar{Z}(r, f)$ the counting function of zeros of $f$ without multiplicity: $\bar{Z}(r, f)=\overline{\omega_{0}}(f) \log r+\sum_{n=1}^{\sigma(r)}\left(\log r-\log \left|a_{n}\right|\right)$ and so, $\bar{Z}(r, f)$ is called the counting function of zeros of $f$ in $d(0, r)$ ignoring multiplicity. 
In the same way, we denote by $\tau(r)$ the number of distinct poles of $f$ in $d(0, r)$ and then, considering the finite sequence $\left(b_{n}\right), 1 \leq n \leq \tau(r)$ of poles of $f$ in $d(0, r)$, with respective multiplicity order $t_{n}$, we put

$N(r, f)=\max \left(-\omega_{0}(f), 0\right) \log r+\sum_{n=1}^{\tau(r)} t_{n}\left(\log r-\log \left|b_{n}\right|\right)$ and then $N(r, f)$ is called the counting function of the poles of $f$, counting multiplicity

Next, in order to define the counting function of poles of $f$ without multiplicity, we put $\overline{\overline{\omega_{0}}}(f)=0$ if $\omega_{0}(f) \geq 0$ and $\overline{\overline{\omega_{0}}}(f)=1$ if $\omega_{0}(f) \leq-1$ and we set

$\bar{N}(r, f)=\overline{\overline{\omega_{0}}}(f) \log r+\sum_{n=1}^{\tau(r)}\left(\log r-\log \left|b_{n}\right|\right)$ and then $\bar{N}(r, f)$ is called the counting function of the poles of $f$, ignoring multiplicity

Now we can define the Nevanlinna function $T(r, f)$ in $I$ or $J$ as $T(r, f)=\max (Z(r, f), N(r, f))$ and the function $T(r, f)$ is called characteristic function of $f$ or Nevanlinna of $f$.

Finally, if $S$ is a subset of $\mathbb{K}$ we will denote by $Z_{0}^{S}\left(r, f^{\prime}\right)$ the counting function of zeros of $f^{\prime}$, excluding those which are zeros of $f-a$ for any $a \in S$.

Remark: If we change the origin, the functions $Z, N, T$ are not changed, up to an additive constant.

In a $p$-adic field such as $\mathbb{K}$, the first Main Theorem is almost immediate:

Theorem A: Let $f \in \mathcal{M}(\mathbb{K})$ (resp. $f \in \mathcal{M}\left(d\left(0, R^{-}\right)\right)$) have no zero and no pole at 0 . Then $\log (|f|(r))=\Psi(f, \log r)=\log (|f(0)|)+Z(r, f)-N(r, f)$.

Corollary A.1: Let $f, g \in \mathcal{M}(\mathbb{K})$ (resp. $f, g \in \mathcal{M}\left(d\left(0, R^{-}\right)\right)$). Then $Z(r, f g) \leq$ $Z(r, f)+Z(r, g), N(r, f g) \leq N(r, f)+N(r, g), T(r, f g) \leq T(r, f)+T(r, g), T(r, f+g) \leq$ $\left.T(r, f)+T(r, g)+O(1), T(r, c f)=T(r, f) \forall c \in \mathbb{K}^{*}, T\left(r, \frac{1}{f}\right)=T(r, f)\right)$.

If $f, g \in \mathcal{A}(\mathbb{K})$ (resp. if $f, g \in \mathcal{A}\left(d\left(0, R^{-}\right)\right)$), then $Z(r, f g)=Z(r, f)+Z(r, g), T(r, f)=$ $Z(r, f)), T(r, f g)=T(r, f)+T(r, g)+O(1)$ and $T(r, f+g) \leq \max (T(r, f), T(r, g))$.

We can now state the famous p-adic Second Main Theorem:

Theorem B: Let $\alpha_{1}, \ldots, \alpha_{q} \in \mathbb{K}$, with $q \geq 2$, let $S=\left\{\alpha_{1}, \ldots, \alpha_{q}\right\}$ and let $f \in \mathcal{M}(\mathbb{K})$ (resp. $f \in \mathcal{M}_{u}\left(d\left(0, R^{-}\right)\right)$). Then

$(q-1) T(r, f) \leq \sum_{j=1}^{q} \bar{Z}\left(r, f-\alpha_{j}\right)+\bar{N}(r, f)-Z_{0}^{S}\left(r, f^{\prime}\right)-\log r+O(1) \quad \forall r \in I \quad$ (resp. $\left.\forall r \in J\right)$.

Definitions and notation: For each $f \in \mathcal{M}(\mathbb{K})$ (resp. $f \in \mathcal{M}\left(d\left(a, R^{-}\right)\right)$) we denote by $\mathcal{M}_{f}(\mathbb{K}),\left(\operatorname{resp} . \mathcal{M}_{f}\left(d\left(a, R^{-}\right)\right)\right)$the set of functions $h \in \mathcal{M}(\mathbb{K}),\left(\operatorname{resp} . h \in \mathcal{M}\left(d\left(a, R^{-}\right)\right)\right)$ such that $T(r, h)=o(T(r, f))$ when $r$ tends to $+\infty$ (resp. when $r$ tends to $R$ ). Similarly, 
if $f \in \mathcal{A}(\mathbb{K})$ (resp. $f \in \mathcal{A}\left(d\left(a, R^{-}\right)\right)$) we shall denote by $\mathcal{A}_{f}(\mathbb{K})$ (resp. $\left.\mathcal{A}_{f}\left(d\left(a, R^{-}\right)\right)\right)$the set $\mathcal{M}_{f}(\mathbb{K}) \cap \mathcal{A}(\mathbb{K}),\left(\operatorname{resp} . \mathcal{M}_{f}\left(d\left(a, R^{-}\right)\right) \cap \mathcal{A}\left(d\left(a, R^{-}\right)\right)\right)$.

The elements of $\mathcal{M}_{f}(\mathbb{K})$ (resp. $\mathcal{M}_{f}\left(d\left(a, R^{-}\right)\right)$) are called small functions with respect to $f$. Similarly, if $f \in \mathcal{A}(\mathbb{K})$ (resp. $f \in \mathcal{A}\left(d\left(a, R^{-}\right)\right)$) the elements of $\mathcal{A}_{f}(\mathbb{K})$ (resp. $\left.\mathcal{A}_{f}\left(d\left(a, R^{-}\right)\right)\right)$are called small functions with respect to $f$.

According to classical results [11], [18], we have the following Theorem C:

Theorem C: Let $f \in \mathcal{A}(\mathbb{K})$ (resp. $f \in \mathcal{A}_{u}\left(d\left(0, R^{-}\right)\right.$)) and let $w_{1}, w_{2} \in \mathcal{A}_{f}(\mathbb{K})$ (resp. $\left.w_{1}, w_{2} \in \mathcal{A}_{f}\left(d\left(0, R^{-}\right)\right)\right)$be distinct. Then $T(r, f) \leq \bar{Z}\left(r, f-w_{1}\right)+\bar{Z}\left(r, f-w_{2}\right)+$ $o(T(r, f)))$.

Definitions and notation: Given $f, g \in \mathcal{M}\left(d\left(0, R^{-}\right)\right)$, we denote by $W(f, g)$ the Wronskian of $f$ and $g$ i.e. $f^{\prime} g-f g^{\prime}$.

In [5], the following results are proven:

Theorem D: $\quad$ Let $f, g \in \mathcal{A}(\mathbb{K})$ be such that $W(f, g)$ is a non-identically zero polynomial. Then both $f, g$ are polynomials.

Notation: Let $f \in \mathcal{A}(\mathbb{K})$. We can factorize $f$ in the form $\bar{f} \tilde{f}$ where the zeros of $\bar{f}$ are the distinct zeros of $f$ each with order 1 . Moreover, if $f(0) \neq 0$ we will take $\bar{f}$ such that $\bar{f}(0)=1$.

Theorem E: Let $f \in \mathcal{M}(\mathbb{K})$ have finitely many multiple poles, such that for certain $b \in \mathbb{K}, f^{\prime}-b$ has finitely many zeros. Then $f$ belongs to $\mathbb{K}(x)$.

Notation: Let $f \in \mathcal{M}\left(d\left(0, R^{-}\right)\right)$. For each $\left.r \in\right] 0, R[$, we denote by $\zeta(r, f)$ the number of zeros of $f$ in $d(0, r)$, taking multiplicity into account and set $\tau(r, f)=\zeta\left(r, \frac{1}{f}\right)$. Similarly, we denote by $\beta(r, f)$ the number of multiple zeros of $f$ in $d(0, r)$, each counted with its multiplicity and we set $\gamma(r, f)=\beta\left(r, \frac{1}{f}\right)$.

Theorem F: Let $f \in \mathcal{M}(\mathbb{K})$ be such that for some $c, d \in] 0,+\infty[, \gamma(r, f)$ satisfies $\gamma(r, f) \leq c r^{d}$ in $\left[1,+\infty\left[\right.\right.$. If $f^{\prime}$ has finitely many zeros, then $f \in \mathbb{K}(x)$.

Corollary F.1: Let $f$ be a meromorphic function on $\mathbb{I K}$ such that, for some $c, d \in] 0,+\infty[$, $\gamma(r, f)$ satisfies $\gamma(r, f) \leq c r^{d}$ in $\left[1,+\infty\left[\right.\right.$. If for some $b \in \mathbb{K} f^{\prime}-b$ has finitely many zeros, then $f$ is a rational function.

\section{First results [17]}

We will now prove together the following Theorems 1 and 2 . 
Theorem 1: Let $f \in \mathcal{M}(\mathbb{K}) \backslash \mathbb{K}(x)$ satisfy $\limsup _{r \rightarrow \infty}|f|(r)>0$ and let $b \in \mathbb{K}^{*}$. Let $m \in \mathbb{N}^{*}$ be $\geq 3$. Then $f^{\prime}+b f^{m}$ has infinitely many zeros that are not zeros of $f$.

Theorem 2: Let $f \in \mathcal{M}_{u}\left(d\left(a, R^{-}\right)\right)$satisfy $\limsup _{r \rightarrow R}|f|(r)=+\infty$ and let $b \in \mathbb{K}^{*}$. Let $m \in \mathbb{N}^{*}$ be $\geq 3$. Then $f^{\prime}+b f^{m}$ has infinitely many zeros that are not zeros of $f$.

Proof : Without loss of generality, we can assume $b=1$ and when $f \in \mathcal{M}\left(d\left(a, R^{-}\right)\right)$, we may assume $a=0$. By hypotheses, there exists a sequence of intervals $\left[r_{n}^{\prime}, r_{n}^{\prime \prime}\right]$ such that $\lim _{n \rightarrow+\infty} r_{n}^{\prime}=+\infty$ (resp. $\lim _{n \rightarrow+\infty} r_{n}^{\prime}=\lim _{n \rightarrow+\infty} r_{n}^{\prime \prime}=R$ ) and such that, putting $J=\bigcup_{n \in \mathbb{N}}\left[r_{n}^{\prime}, r_{n}^{\prime \prime}\right]$, we have $\liminf _{\substack{r \rightarrow \infty \\ r \in J}}|f|(r)>0$ (resp. $\left.\lim _{\substack{r \rightarrow R^{-} \\ r \in J}}|f|(r)=+\infty\right)$.

Suppose first we assume the hypothesis of Theorem 1. Let $M=\frac{\liminf _{r \rightarrow+\infty}|f|(r)}{2}$. We will prove that there exists $t>0$ such that $\left|f^{\prime}+f^{m}\right|(r)=\left|f^{m}\right|(r) \forall r \in J \cap[t,+\infty[$. We know that $\left|f^{\prime}\right|(r) \leq \frac{|f|(r)}{r}$. Consequently, when $r$ lies in $J$, there exists $s>0$ such that $|f|(r) \geq M \forall r \in[s,+\infty[\cap J$.

$$
(|f|(r))^{m} \geq|f|(r) M^{m-1} \geq r\left|f^{\prime}\right|(r) M^{m-1} .
$$

Next, when $r$ is big enough, $r M^{m-1}$ is greater than 1 , hence $(|f|(r))^{m}>\left|f^{\prime}\right|(r)$. Thus there exists $t \geq s$ such that $(|f|(r))^{m}>\left|f^{\prime}\right|(r) \forall r \in J \cap\left[t,+\infty\left[\right.\right.$. Let $J^{\prime}=J \cap[t,+\infty[$. And hence we have $\left|f^{\prime}+f^{m}\right|(r)=\left|f^{m}\right|(r) \forall r \in J^{\prime}$.

Suppose now that we assume the hypothesis of Theorem 2 We have $\left|f^{\prime}\right|(r) \leq \frac{|f|(r)}{r} \leq \frac{|f|(r)}{R}$. Set $B=\frac{1}{R}$. Then we have

$$
(|f|(r))^{m} \geq B\left|f^{\prime}\right|(r)(|f|(r))^{m-1}
$$

Now, when $r$ is close enough to $R, r \in J, B|f(x)|^{m-1}$ is strictly greater than 1 , hence $(|f|(r))^{m}>\left|f^{\prime}\right|(r)$. Thus there exists $t>0$ such that $(|f|(r))^{m}>\left|f^{\prime}\right|(r) \forall r \in[t,+\infty[\cap J$. We can set again $J^{\prime}=J \cap\left[t, R\left[\right.\right.$ and then we have $\left|f^{\prime}+f^{m}\right|(r)=\left|f^{m}\right|(r) \forall r \in J^{\prime}$

We can now conclude in both theorems 1 and 2 . For each $n \in \mathbb{N}$, let $q_{n}$ be the number of zeros of $f$ in $d\left(0, r_{n}^{\prime \prime}\right)$. Suppose the sequence $\left(q_{n}\right)_{n \in \mathbb{N}}$ is bounded. Then, $f$ has finitely many zeros, hence it is of the form $\frac{P}{h}$ with $P \in \mathbb{K}[x]$ and $h \in \mathcal{A}_{u}(d(0, R))$. Consequently, we have $\lim _{r \rightarrow+\infty}|f|(r)=0$, a contradiction to the hypothesis in both theorems. Therefore, the sequence $\left(q_{n}\right)_{n \in \mathbb{N}}$ which is increasing by definition, tends to $+\infty$. Now, in each Theorems 1 and 2 we may apply Lemma 3 showing that $f^{\prime}+f^{m}$ has infinitely many zeros that are not zeros of $f$.

Consider now the case $m=1$. We can have a better conclusion in $\mathcal{M}(\mathbb{K})$. 
Theorem 3: Let $f \in \mathcal{M}(\mathbb{K}) \backslash \mathbb{K}(x)$. For each $b \in \mathbb{K}^{*}, f^{\prime}+b f$ has infinitely many zeros that are not zeros of $f$.

Proof: Without loss of generality, we can assume again $b=1$. We have $\left|f^{\prime}\right|(r)<|f|(r)$ when $r$ is big enough and hence $\left|f^{\prime}+f\right|(r)=|f|(r)$ in an interval $I=[s,+\infty$ [. Suppose first that $f$ has infinitely many zeros. We can then apply Lemma 3 and get the conclusion.

Suppose now that $f$ has finitely many zeros. Then $f$ has infinitely many poles $c_{n}$ of respective order $t_{n}$. Since $\mathbb{K}$ has characteristic zero, $f^{\prime}$ admits each $c_{n}$ as a pole of order $t_{n}+1$ and similarly, $f^{\prime}+f$ also admits each $c_{n}$ as a pole of order $t_{n}+1$. Thus, we have $N\left(r, f^{\prime}+f\right)=N(r, f)+\bar{N}(r, f)$. But since $\left|f^{\prime}+f\right|(r)=|f|(r)$ holds in $I$, we have $\Psi\left(f^{\prime}+f, \log r\right)=\Psi(f, \log r) \forall r \in I$ hence, by Lemma $2, \nu\left(f^{\prime}+f, \log r\right)=\nu(f, \log r) \forall r \in I$ and hence $Z\left(r, f^{\prime}+f\right)-N\left(r, f^{\prime}+f\right)=Z(r, f)-N(r, f)$, therefore $Z\left(r, f^{\prime}+f\right)-(N(r, f)+$ $\bar{N}(r, f))=Z(r, f)-N(r, f)$ and hence $Z\left(r, f^{\prime}+f\right)=Z(r, f)+\bar{N}(r, f)$. Since we have supposed that $f$ has finitely many zeros and since $f$ has infinitely many poles, $f^{\prime}+f$ has infinitely many zeros and all but finitely many are not zeros of $f$.

Theorem 4: Let $f \in \mathcal{A}(\mathbb{K}) \backslash \mathbb{K}(x)$ (resp. let $a \in \mathbb{K}$, let $R \in] 0,+\infty[$ and let $f \in$ $\mathcal{A}_{u}\left(d a\left(, R^{)}\right)\right)$. For each $b \in \mathbb{K}^{*}, f^{\prime}+b f^{2}$ has infinitely many zeros that are not zeros of $f$.

Proof: Without loss of generality, we can assume $b=1$ and $a=0$. Clearly, when $r$ is big enough, in ]0, $+\infty$ [ (resp. in ]0, $R\left[\right.$ ), we have $\left|f^{\prime}+f^{2}\right|(r)=\left|f^{2}\right|(r)$ therefore $f^{2}$ and $f^{\prime}+f^{2}$ have the same number of zeros in $C(0, r)$. Let $\alpha \in C(0, r)$ be a zero of $f$ of order $q$. When $r$ is big enough, it is a zero of order $2 q$ for $f^{2}$ and it is a zero of order $q-1$ for $f^{\prime}+f^{2}$. Consequently, $f^{\prime}+f^{2}$ has at least $q+1$ zero in $C(0, r)$ that are not zeros of $f$ (taking multiplicity into account). This is true for every such zeros of $f$ an hence $f^{\prime}+f^{2}$ has infinitely many zeros that are not zeros of $f$.

Corollary 4.1: Let $m \in \mathbb{N}$ be $\geq 1$, let $f \in \mathcal{A}(\mathbb{K}) \backslash \mathbb{K}(x)$. For each $b \in \mathbb{K}^{*}, f^{\prime}+b f^{m}$ has infinitely many zeros that are not zeros of $f$.

Corollary 4.2: Let $m \in \mathbb{N}$ be $\geq 2$, let $a \in \mathbb{K}$, let $R \in] 0,+\infty\left[\right.$ and let $f \in \mathcal{A}_{u}\left(d a\left(, R^{-}\right)\right)$. For each $b \in \mathbb{K}^{*}, f^{\prime}+b f^{m}$ has infinitely many zeros that are not zeros of $f$.

Theorem 5 is given in [4]:

Theorem 5: Let $f \in \mathcal{M}(\mathbb{K}) \backslash \mathbb{K}(x)$ (resp. let $a \in \mathbb{K}$ and $R \in \mathbb{R}_{+}^{*}$ and let $f \in$ $\left.\mathcal{M}\left(d\left(a, R^{-}\right)\right)\right)$and let $m \in \mathbb{N}$. If $m \geq 5$ then for each $b \in \mathbb{K}^{*}, f^{\prime}+b f^{m}$ has infinitely many zeros that are not zeros of $f$.

If $m=4$, if $f \in \mathcal{M}(\mathbb{K}) \backslash \mathbb{K}(x)$ and if $f$ admits at least $s$ multiple zeros and at least $t$ multiple poles, then $f^{\prime}+b f^{4}$ admits a number of zeros that are not zeros of $f$ (taken account of multiplicity) which is strictly superior to $\frac{s+t}{2}$.

Proof: We know that the zeros of $f^{\prime}+b f^{m}$ in $\mathbb{K}$ are the same as in a spherically complete algebraically closed extension $\widehat{\mathbb{K}}$ of $\mathbb{K}$. So, for simplicity, we can suppose that the field $\mathbb{K}$ 
is spherically complete without loss of generality. We can also suppose that $b=1$. Then if $f \in \mathcal{M}(\mathbb{K}) \backslash \mathbb{K}(x)$ we can obviously we can write $f=\frac{h}{l}$ with $h, l \in \mathcal{A}(\mathbb{K})$, having no common zeros and if $f \in \mathcal{M}\left(d\left(a, R^{-}\right)\right)$, since $\mathbb{K}$ is spherically complete, we can write $f=\frac{h}{l}$ with $h, l \in \mathcal{A}\left(d\left(a, R^{-}\right)\right)$, having no common zeros again.

Let $g=\frac{1}{f}$ and let $n=m-2$. So, by Lemma 1 , the problem is reduced to show that $g^{\prime} g^{n}-1$ has infinitely many zeros. Then, $g^{\prime} g^{n}-1=\frac{\left(l^{\prime} h-h l^{\prime}\right) l^{n}-h^{n+2}}{h^{n+2}}$ and since $h, l$ have no common bzeros, this is of the form $\frac{P}{h^{n+2}}$ where $P$ is a polynomial of degree $q$. Now, set $F=\left(l^{\prime} h-h l^{\prime}\right) l^{n}$. Applying Theorem $C$ to $F$ we have

(1) $T(r, F)=Z(r, F)+O(1) \leq \bar{Z}(r, F)+\bar{Z}(r, F-P)+T(r, P)+O(1)$. By (1) we derive $Z\left(r, l^{\prime} h-h^{\prime} l\right)+n Z(r, l) \leq \bar{Z}\left(r, l^{\prime} h-h^{\prime} l\right)+\bar{Z}(r, l)+\bar{Z}(r, F-P)+T(r, P)+O(1)$. Actually, $\bar{Z}(r, F-P)=\bar{Z}(r, h)$, hence $n Z(r, l) \leq \bar{Z}(r, l)+\bar{Z}(r, h)+T(r, P)+O(1)$ and hence $(n-1) Z(r, l) \leq Z(r, h)+T(P)+O(1)$. But since $T(r, P)=q \log r+O(1)$, we have

(2) $\quad(n-1) Z(r, l) \leq Z(r, h)+q \log r+O(1)$

Now, consider the hypothesis $f \in \mathcal{M}(\mathbb{K})$. By Theorem 1, if $\liminf _{r \rightarrow+\infty}|f|(r)>0$ i.e. if $\liminf _{r \rightarrow+\infty} Z(r, f)-N(r, f)>-\infty$ the claim is proved. Consequently, if the claim is not true, we can assume

(3) $\liminf _{r \rightarrow+\infty} Z(r, f)-N(r, f)=-\infty$

But we see that (3) is impossible whenever $n \geq 3$, i.e. $m \geq 5$.

Now, suppose $m=4$.i.e $n=2$. More precisely $\bar{Z}(r, l) \leq Z(r, l)-\frac{s \log r}{2}$ and $\bar{Z}(r, h) \leq Z(r, h)-\frac{t \log r}{2}$, so by Relation (1) we have

(4) $(n-1) Z(r, l) \leq Z(r, h)+\left(q-\frac{s+t}{2}\right) \log r+O(1)$.

Then Relation (3) implies $q-\frac{s+t}{2}>0$ and hence $f^{\prime} f^{n}$ admits a number of zeros strictly superior to $\frac{s+t}{2}$.

Now, suppose that $f \in \mathcal{M}\left(d\left(0, R^{-}\right)\right)$. By Theorem 2, if $\lim _{r \rightarrow R^{-}}|f|(r)=+\infty$ i.e. if $\liminf _{r \rightarrow R^{-}} Z(r, f)-N(r, f)=+\infty$ the claim is proved. Consequently, if the claim is not true, we can assume

(5) $\liminf _{r \rightarrow R^{-}} Z(r, f)-N(r, f)<+\infty$.

But by (2), we see that (5) is impossible whenever $n \geq 3$ i.e. $m \geq 5$.

Corollary 4.1: Let $f \in \mathcal{M}(\mathbb{K}) \backslash \mathbb{K}(x)$. Then for each $n \geq 3 f^{\prime} f^{n}$ has infinitely many zeros that are not zeros of $f$. 
Corollary 4.2: Let $f \in \mathcal{M}(\mathbb{K}) \backslash \mathbb{K}(x)$ have $s$ multiple zeros and $t$ multiple poles. Let $b \in \mathbb{K}^{*}$. Then $f^{\prime}+b f^{4}$ has at least $s+t+1$ zeros taking account of multiplicity. Particularly, if $f$ has infinitely many multiple zeros or poles, then $f^{\prime}+b f^{4}$ has infinitely many zeros that are not zeros of $f$.

Corollary 4.3: Let $f \in \mathcal{M}(\mathbb{K}) \backslash \mathbb{K}(x)$ have $s$ multiple zeros and $t$ multiple poles. Given $b \in \mathbb{K}^{*}$, If $f$ has infinitely many multiple zeros or poles, then $f^{\prime} f^{2}-b$ has infinitely many zeros.

Case $n=2, m=4$

We will now thorougly examine the situation when $m=4$ i.e. $n=2$, as made in [9]. This requires several basic lemmas.

Lemma 4: Let $f \in \mathcal{M}(\mathbb{K})$ be transcendental and such that $f^{\prime}$ has finitely many multiple zeros. Then $\frac{f^{\prime \prime} f}{\left(f^{\prime}\right)^{2}}$ has no quasi-exceptional value.

Proof: Let $g=\frac{f}{f^{\prime}}$. A pole of $g$ is a zero of $f^{\prime}$, hence by hypothesis, $g$ has finitely many multiple poles. Consequently, by Theorem E, $g^{\prime}$ has no quasi-exceptional value. And hence neither has $1-g^{\prime}$. But $g^{\prime}=\frac{\left(f^{\prime}\right)^{2}-f^{\prime \prime} f}{\left(f^{\prime}\right)^{2}}=1-\frac{f^{\prime \prime} f}{\left(f^{\prime}\right)^{2}}$. Therefore $\frac{f^{\prime \prime} f}{\left(f^{\prime}\right)^{2}}$ has no quasi-exceptional value.

Lemma 5: Let $f \in \mathcal{M}(\mathbb{K})$ be transcendental and have finitely many multiple zeros. Then $f^{\prime \prime} f+2\left(f^{\prime}\right)^{2}$ has infinitely many zeros that are not zeros of $f$.

Proof: Suppose first that $f^{\prime}$ has infinitely many multiple zeros. Since $f$ has finitely many multiple zeros, the zeros of $f^{\prime}$ are not zeros of $f$ except at most finitely many. Hence $f^{\prime}$ has infinitely many multiple zeros that are not zeros of $f$. And then, they are zeros of $f^{\prime \prime}$, hence of $f^{\prime \prime} f+2\left(f^{\prime}\right)^{2}$, which proves the statement.

So we are now led to assume that $f^{\prime}$ has finitely many multiple zeros. By Lemma 4 $\frac{f^{\prime \prime} f+2\left(f^{\prime}\right)^{2}}{\left(f^{\prime}\right)^{2}}$ has infinitely many zeros. Let $c \in \mathbb{K}$ be a pole of order $q$ of $f$. Without loss of generality, we can suppose $c=0$. The beginning of the Laurent developpement of $f$ at 0 is of the form $\frac{a_{-q}}{x^{q}}+\frac{\varphi(x)}{x^{q-1}}$ whereas $\varphi \in \mathcal{M}(\mathbb{K})$ has no pole at 0 . Consequently, $\frac{f^{\prime \prime} f+2\left(f^{\prime}\right)^{2}}{\left(f^{\prime}\right)^{2}}$ is of the form

$$
\frac{\left(a_{-q}\right)^{2}\left(3 q^{2}+q\right)+x \phi(x)}{\left(a_{-q}\right)^{2}\left(q^{2}\right)+x \psi(x)}
$$

whereas $\phi, \psi \in \mathcal{M}(\mathbb{K})$ have no pole at 0 . So, the function $\frac{f^{\prime \prime} f+2\left(f^{\prime}\right)^{2}}{\left(f^{\prime}\right)^{2}}$ has no zero at 0 . 
Therefore, each zero of $\frac{f^{\prime \prime} f+2\left(f^{\prime}\right)^{2}}{\left(f^{\prime}\right)^{2}}$ is a zero of $f^{\prime \prime} f+2\left(f^{\prime}\right)^{2}$ and hence $f^{\prime \prime} f+2\left(f^{\prime}\right)^{2}$ has infinitely many zeros.

Now, let us show that the zeros of $f^{\prime \prime} f+2\left(f^{\prime}\right)^{2}$ are not zeros of $f$, except maybe finitely many. Let $c$ be a zero of $f^{\prime \prime} f+2\left(f^{\prime}\right)^{2}$ and suppose that $c$ is a zero of $f$. Then, it is a zero of $f^{\prime}$ and hence it is a multiple zero of $f$. But by hypotheses, $f$ has finitely many multiple zeros, hence the zeros of $f^{\prime \prime} f+2\left(f^{\prime}\right)^{2}$ are not zeros of $f$, except at most finitely many. That finishes proving the claim.

Lemma 6: Let $f \in \mathcal{M}(\mathbb{K})$ be transcendental and let $b \in \mathbb{K}^{*}$ be such that $f^{2} f^{\prime}-b$ has finitely many zeros. Then, $N(r, f) \leq Z(r, f)+O(1)$.

Proof: Let $F=f^{2} f^{\prime}$. Since $F-b$ is transcendental and has finitely many zeros, it is of the form $\frac{P(x)}{h(x)}$ with $h \in \mathcal{A}(\mathbb{K}) \backslash \mathbb{K}[x]$. Consequently, $|F|(r)$ is a constant when $r$ is big enough and therefore, by Theorem A we have $Z(r, F)=N(r, F)+O(1)$ when $r$ is big enough. Now, $Z(r, F)=2 Z(r, f)+Z\left(r, f^{\prime}\right)$ and, by Theorem A $Z\left(r, f^{\prime}\right) \leq Z(r, f)+\bar{N}(r, f)-\log r+$ $O(1)$. On the other hand, by Theorem A again, we have $N(r, F)=3 N(r, f)+\bar{N}(r, f)$. Consequently, $3 N(r, f)+\bar{N}(r, f) \leq 3 Z(r, f)+\bar{N}(r, f)-\log r+O(1)$, which proves the claim.

Theorem 6: Let $f \in \mathcal{M}(\mathbb{K}) \backslash \mathbb{K}(x)$ Then for each $b \in \mathbb{K}^{*}, f^{\prime} f^{2}-b$ has infinitely many zeros.

Proof: Let $b \in \mathbb{K}$ and suppose that the claim is wrong, i.e. $f^{2} f^{\prime}-b$ has $q$ zeros, taking multiplicity into account. By Theorem 5 , we may assume that $f$ has finitely many multiple zeros and finitely multiple poles. Set $F=f^{2} f^{\prime}$. Then $F^{\prime}=f\left(f^{\prime \prime} f+2\left(f^{\prime}\right)^{2}\right)$. By Lemma $5, f^{\prime \prime} f+2\left(f^{\prime}\right)^{2}$ has infinitely many zeros that are not zeros of $f$. Consequently, $F^{\prime}$ admits for zeros: the zeros of $f$ and the zeros of $f^{\prime \prime} f+2\left(f^{\prime}\right)^{2}$. And by Lemma 4 , there exists a sequence of zeros of $f^{\prime \prime} f+2\left(f^{\prime}\right)^{2}$ that are not zeros of $f$.

Let $S=\{0, b\}$ and let $Z_{0}^{S}\left(r, F^{\prime}\right)$ be the counting function of zeros of $F^{\prime}$ when $F(x)$ is different from 0 and $b$. Since $F-b$ has finitely many zeros, the zeros $c$ of $F^{\prime}$ which are not zeros of $f$ cannot satisfy $F(c)=b$ except at most finitely many. Consequently, there are infinitely many zeros of $F^{\prime}$ counted by the counting function $Z_{0}^{S}\left(r, F^{\prime}\right)$ and hence for every fixed integer $t \in \mathbb{N}$, we have

$$
Z_{0}^{S}\left(r, F^{\prime}\right) \geq t \log r+O(1)
$$

Let us apply Theorem B to $F$. We have

$$
T(r, F) \leq \bar{Z}(r, F)+\bar{Z}(r, F-b)+\bar{N}(r, F)-Z_{0}^{S}\left(r, F^{\prime}\right)-\log (r)+O(1) .
$$

Now, we have

$$
\bar{Z}(r, F) \leq Z(r, f)+Z\left(r, f^{\prime}\right)
$$




$$
\bar{N}(r, F)=\bar{N}(r, f)
$$

and since the number of zeros of $F-b$ is $q$, taking multiplicity into account,

$$
\bar{Z}(r, F-b) \leq q \log r+O(1)
$$

Consequently, by (2), (3), (4), (5) we obtain

$$
T(r, F) \leq Z(r, f)+Z\left(r, f^{\prime}\right)+\bar{N}(r, f)-Z_{0}^{S}\left(r, F^{\prime}\right)+(q-1) \log r+O(1)
$$

On the other hand, by construction, $T(r, F) \geq Z(r, F)=2 Z(r, f)+Z\left(r, f^{\prime}\right)$ hence by (6) we obtain (7):

$$
Z(r, f) \leq \bar{N}(r, f)-Z_{0}^{S}\left(r, F^{\prime}\right)+(q-1) \log r+O(1)
$$

Now, by Lemma 6 , we have $N(r, f) \leq Z(r, f)+O(1)$ hence by (7) we obtain $0 \leq$ $(q-1) \log r-Z_{0}^{S}\left(r, F^{\prime}\right)+O(1)$ and hence by (1), fixing $t>q-1$ we can derive $0 \leq$ $(q-1) \log r-t \log r+O(1)$, a contradiction. That finishes the proof of Theorem 6 .

By Lemma 1, Theorems 5 and 6 we can now state the general result on the $p$-adic Hayman conjecture:

Corollary 6.1: Let $f \in \mathcal{M}(\mathbb{K})$ be transcendental. Then for every $n \in \mathbb{N}, n \geq 2$, for every $b \in \mathbb{K}^{*}, f^{2} f^{\prime}-b$ has infinitely many zeros and for every $m \geq 4, f^{\prime}+b f^{m}$ has infinitely many zeros that are not zeros of $f$.

Case $n=1, m=3$

Concerning the case $m=3$ i.e. $n=1$ which remains unsolved, Corollary 6.1 has an immediate application to the conjecture with additional hypotheses [2].

Theorem 7: Let $f \in \mathcal{M}(\mathbb{K})$. Suppose that there exists $c, d \in] 0,+\infty[$, such that $\tau(r, f) \leq$ ${ }^{c} r^{d} \forall r \in\left[1,+\infty\left[\right.\right.$. If $f^{\prime} f^{n}-b$ has has finitely many zeros for some $b \in \mathbb{K}$, with $n \in \mathbb{N}$, then $f \in \mathbb{K}(x)$.

Proof: Suppose $f$ is transcendental. By hypothesis, $f^{n+1}$ satisfies $\zeta\left(r, \frac{1}{f^{n+1}}\right)=\tau\left(r, f^{n+1}\right) \leq$ $c(n+1) r^{d} \forall r \in\left[1,+\infty\left[\right.\right.$ hence by Corollary $6.1, f^{\prime} f^{n}$ has no quasi-exceptional value.

Theorem 7 may be writen in another way:

Corollary 7.1: Let $f \in \mathcal{M}(\mathbb{K}) \backslash \mathbb{K}(x)$. Suppose that there exists $c, d \in] 0,+\infty[$, such that $\zeta(r, f) \leq c r^{d} \forall r \in\left[1,+\infty\left[\right.\right.$. Then for all $m \in \mathbb{N}, m \geq 3$ and for all $b \in \mathbb{K}, f^{\prime}-b f^{m}$ admits infinitely many zeros that are not zeros of $f$. 
Proof: We set $g=\frac{1}{f}$. Then by Theorem $7 g^{\prime} g^{m-2}$ has no quasi-exceptional value. Consequently, given $b \in \mathbb{K}^{*}, g^{\prime} g^{m-2}+b$ has infinitely many zeros and hence $f^{\prime}-b f^{m}$ has infinitely many zeros that are not zeros of $f$. Next, if $b=0$, by Theorem $\mathrm{F}$, $f^{\prime}$ has infinitely many zeros.

Theorem 8: Let $f \in \mathcal{M}(\mathbb{K})$. Suppose that there exists $c, d \in] 0,+\infty[$, such that $\beta(r, f) \leq$ $c r^{d} \forall r \in\left[1,+\infty\left[\right.\right.$. Then, for all $b \in \mathbb{K}, \frac{f^{\prime}}{f^{2}}-b$ has infinitely many zeros.

Proof: Set $g=\frac{1}{f}$ again. Since the poles of $g$ are the zeros of $f$, we have $\gamma(r, g) \leq c r^{d}$. Consequently, by Corollary F.1, $g^{\prime}$ has no quasi-exceptional value.

Remark: Using Theorem 8 to study the zeros of $f^{\prime}-b f^{2}$ that are not zeros of $f$ is not so immediate, as we will see below because of residues of $f$ at poles of order 1 . Of course, if $\frac{1}{f}$ is an affine function, $f^{\prime}+f^{2}$ has no zeros, except if it is identically zero. And if it is not identically zero, the residue at the pole is not 1 in the general case.

Case $n=0$ i.e. $m=2$

As we noticed at the beginning, due to the counter-example provided by the function tan, the case $n=0$ has no solution in $\mathbb{C}$. However, we can notice certain conclusions.

Lemma 7: Let $f=\frac{h}{l} \in \mathcal{M}(\mathbb{K})$ with $h, l \in \mathcal{A}(\mathbb{K})$ having no common zero, let $b \in \mathbb{K}^{*}$ and let $a \in \mathbb{K}$ be a zero of $h^{\prime} l-h l^{\prime}+b h^{2}$ that is not a zero of $f^{\prime}+b f^{2}$. Then a is a pole of order 1 of $f$ and $\operatorname{res}(f, a)=\frac{1}{b}$.

Proof: Clearly, if $l(a) \neq 0, a$ is a zero of $f^{\prime}+b f^{2}$. Hence, a zero $a$ of $h^{\prime} l-h l^{\prime}+b h^{2}$ that is not a zero of $f^{\prime}+b f^{2}$ is a pole of $f$. Now, when $l(a)=0$, we have $h(a) \neq 0$ hence $l^{\prime}(a)=b h(a) \neq 0$ and therefore $a$ is a pole of order 1 of $f$ such that $\frac{h(a)}{l^{\prime}(a)}=\frac{1}{b}$. But since $a$ is a pole of order 1 , we have $\operatorname{res}(f, a)=\frac{h(a)}{l^{\prime}(a)}$, which ends the proof.

Theorem 9 is not a result specific to $p$-adic analysis but it will be useful in Theorem 10.

Theorem 9: Let $f \in \mathcal{M}(\mathbb{K})$, (resp. let $a \in \mathbb{K}$, let $f \in \mathcal{M}\left(d\left(a, R^{-}\right)\right)$), let $b \in \mathbb{K}^{*}$ and let $\alpha \in \mathbb{K}$ (resp. let $\alpha \in d\left(a, R^{-}\right)$) be a point that is not a zero of $f$ and such that the residue of $f$ at $\alpha$ is different from $\frac{1}{b}$. Then $\alpha$ is a zero of $f^{\prime}+b f^{2}$ if and only if it is a zero of $\frac{f^{\prime}}{f^{2}}+b$. Moreover, if it is a zero of both functions, it has the same multiplicity with both. 
Proof: Suppose first $\alpha$ is a zero of $f^{\prime}+b f^{2}$. If $\alpha$ is not a pole of $f$, of course it is a zero of $\frac{f^{\prime}}{f^{2}}+b$ with same multiplicity. Suppose now that $\alpha$ is a pole of $f$ : since it is not a pole of $f^{\prime}+b f^{2}$ it must be a pole of order 1 of $f$. Without loss of generality, we may assume that $\alpha=0$ (resp. $a=\alpha=0$ ). Consider the Laurent series of $f$ at 0 : $f(x)=\frac{a_{-1}}{x}+a_{0}+a_{1} x+x^{2} \phi(x)$ with $\phi \in \mathcal{M}(\mathbb{K})\left(\right.$ resp. $\phi \in \mathcal{M}\left(d\left(0, R^{-}\right)\right)$and $\phi(0) \neq \infty$. Then $f^{\prime}+b f^{2}$ is of the form

$$
f^{\prime}(x)+b f(x)^{2}=\frac{a_{-1}\left(-1+b a_{-1}\right)}{x^{2}}+\frac{2 b a_{0} a_{1}}{x}+a_{1}+b\left(a_{0}^{2}+2 a_{1} a_{-1}\right)+x \xi(x)
$$

with $\xi \in \mathcal{M}(\mathbb{K})$ (resp. $\xi \in \mathcal{M}\left(d\left(0, R^{-}\right)\right)$and $\xi(0) \neq \infty$ and hence, we have $a_{-1}\left(-1+b a_{-1}\right)=0, a_{0} a_{-1}=0, a_{0}^{2}+2 a_{1} a_{-1}=0$. Since by hypothesis $\operatorname{res}(f, \alpha) \neq-\frac{1}{b}$ we have $\left(1+b a_{-1}\right) \neq 0$, hence $a_{-1}=0$, a contradiction. Consequently, every zero of $f^{\prime}+b f^{2}$ that is not a zero of $f$ is a zero of $\frac{f^{\prime}}{f^{2}}+b$ with same multiplicity.

Conversely, suppose now that $\alpha$ is a zero of $\frac{f^{\prime}}{f^{2}}+b$. If $\alpha$ is not a pole of $f$, it is a zero of $f^{\prime}+b f^{2}$, with the same multiplicity, because by hypothesis it is not a zero of $f$. Now suppose that $\alpha$ is a zero of $\frac{f^{\prime}}{f^{2}}+b$ and is a pole of $f$. Clearly, it is a pole of order 1 and again, we may assume that $\alpha=0$.

Consider again the Laurent series of $f$ at $0: f(x)=\frac{a_{-1}}{x}+a_{0}+a_{1} x+x^{2} \phi(x)$ with $\phi \in \mathcal{M}(\mathbb{K})$ and $\phi(0) \neq \infty$. Then

$$
\frac{f^{\prime}}{f^{2}}=\frac{\frac{-a_{-1}}{x^{2}}+a_{1}+x \psi(x)}{\frac{\left(a_{-1}\right)^{2}}{x^{2}}+\frac{2 a_{0} a_{1}}{x}+a_{0}^{2}+2 a_{1} a_{-1}+x \xi(x)}
$$

where both $\psi, \xi \in \mathcal{M}(\mathbb{K})$ have no pole at 0 . Clearly, $\frac{f^{\prime}}{f^{2}}$ is analytic at 0 and its value is $\frac{-1}{a_{-1}}$. But since 0 is a zero of $\frac{f^{\prime}}{f^{2}}+b$, we have $a_{-1}=\frac{1}{b}$, what is excluded by hypothesis. Thus we have proved that every zero of $\frac{f^{\prime}}{f^{2}}+b$ is a zero of $f^{\prime}+b f^{2}$ (that is not a zero of f) with the same multiplicity and this ends the proof of Theorem 9 .

Theorem 10: Let $b \in \mathbb{K}^{*}$ and let $f \in \mathcal{M}(\mathbb{K})$ have finitely many zeros and finitely many residues at its simple poles equal to $\frac{1}{b}$ and be such that $f^{\prime}+b f^{2}$ has finitely many zeros. Then $f$ belongs to $\mathbb{K}(x)$.

Proof: Let $f=\frac{P}{l}$ with $P \in \mathbb{K}[x], l \in \mathcal{A}(\mathbb{K})$ having no common zero with $P$. Then 
$f^{\prime}+b f^{2}=\frac{P^{\prime} l-l^{\prime} P+b P^{2}}{l^{2}}$. By hypothesis, this function has finitely many zeros. Moreover, if $a$ is a zero of $P^{\prime} l-l^{\prime} P+b P^{2}$ but is not a zero of $f^{\prime}+b f^{2}$, then by Lemma 7 it is a pole of order 1 of $f$ such that $\operatorname{res}(f, a)=\frac{1}{b}$. Consequently, $P^{\prime} l-l^{\prime} P+b P^{2}$ has finitely many zeros and hence, we may write $\frac{P^{\prime} l-l^{\prime} P+b P^{2}}{l^{2}}=\frac{Q}{l^{2}}$ with $Q \in \mathbb{K}[x]$, hence $P^{\prime} l-l^{\prime} P=-b P^{2}+Q$. But then, by Theorem $\mathrm{D}, l$ is a polynomial, which ends the proof.

Remark: If $f(x)=\frac{1}{x}$, the function $f^{\prime}+b f^{2}$ has no zero whenever $b \neq 1$.

Theorem 11: Let $f \in \mathcal{M}(\mathbb{K})$ be transcendental and have finitely many zeros of order $\geq 2$ and let $b \in \mathbb{K}$. Then $\frac{f^{\prime}}{f^{2}}+b$ has infinitely many zeros. Moreover, if $b \neq 0$, every zero $\alpha$ of $\frac{f^{\prime}}{f^{2}}+b$ that is not a zero of $f^{\prime}+b f^{2}$ is a pole of $f$ of order 1 such that the residue of $f$ at $\alpha$ is equal to $\frac{1}{b}$.

Proof: Let $g=\frac{f^{\prime}}{f^{2}}+b$. Since all zeros of $f$ are of order 1 except maybe finitely many, $g$ has finitely many poles of order $\geq 3$, hence a primitive $G$ of $g$ has finitely many poles of order $\geq 2$. Consequently, by Theorem E, $g$ has infinitely many zeros.

Now, suppose $b \neq 0$. Let $\alpha$ be a zero of $g$. If $\alpha$ is not a pole of $f$, it is a zero of $f^{\prime}+b f^{2}$ and we can see that it is not a zero of $f$.

Finally, suppose that $\alpha$ is a pole of $f$. Then it must be a pole of order 1 and then, by Lemma 7 , the residue of $f$ at $\alpha$ is $\frac{1}{b}$.

Corollary 11.1: Let $f \in \mathcal{M}(\mathbb{K}) \backslash \mathbb{K}(x)$ have finitely many zeros of order $\geq 2$ and finitely many poles of order 1 and let $b \in \mathbb{K}^{*}$. Then $f^{\prime}+b f^{2}$ has infinitely many zeros that are not zeros of $f$.

Remarks: As noticed above, in Archimedean analysis, the typical example of a meromorphic function $f$ such that $f^{\prime}-f^{2}$ has no zero is $\tan (x)$ and its residue is -1 at each pole of $f$. Here we find the same implication but we can't find an example satisfying such properties.

\section{References}

[1] Bergweiler, W. and Eremenko, A. On the singularities of the inverse to a meromorphic function of nite order, Rev. Mat. Iberoamericana, 11, 355-373 (1995).

[2] Bezivin, J.-P., Boussaf, K., Escassut, A. Zeros of the derivative of a p-adic meromorphic function, Buletin des Sciences Mathématiques 136, 8, 839-847 (2012). 
[3] Boussaf, K. Picard values of p-adic meromorphic functions, p-Adic Numbers Ultrametric Anal. Appl. 2, no. 4, p.285-292 (2010).

[4] Boussaf, K. and Ojeda, J. Value distribution of p-adic meromorphic functions, Bull. Belg. Math. Soc. Simon Stevin, 18, n.4, p. 667-678 (2011).

[5] Boussaf, K., Ojeda, J. and Escassut, A. Zeros of the derivative of a p-adic meromorphic function and applications Bull. Belg. Math. Soc. Simon Stevin 19, n.2, p. 367-372 (2012).

[6] Boutabaa, A. Théorie de Nevanlinna p-adique, Manuscripta Math. 67, p. 251-269 (1990).

[7] Boutabaa, A. and Escassut, A., URS and URSIMS for p-adic meromorphic functions inside a disk, Proc. of the Edinburgh Mathematical Society 44, p. 485-504 (2001).

[8] Escassut, A. Analytic Elements in p-adic Analysis. World Scientific Publishing Co. Pte. Ltd. Singapore, (1995).

[9] Escassut, A. , Ojeda, J. The p-adic Hayman conjecture when $n=2$, Complex variable and elliptic equations.

[10] Hayman W. K., Meromorphic Functions. Oxford University Press, (1975)

[11] Hu, P.C. and Yang, C.C. Meromorphic Functions over non-Archimedean Fields, Kluwer Academic Publishers, (2000).

[12] Hu, P.C. and Yang, C.C. Value Distribution Theory Related to Number Theory Bikhäuser, (2006).

[13] Krasner, M. Prolongement analytique uniforme et multiforme dans les corps valués complets. Les tendances géométriques en algèbre et théorie des nombres, ClermontFerrand, p.94-141 (1964). Centre National de la Recherche Scientifique (1966), (Colloques internationaux de C.N.R.S. Paris, 143).

[14] Lazard, M. Les zéros des fonctions analytiques sur un corps valué complet, IHES, Publications Mathématiques no. 14, pp. 47-75 ( 1962).

[15] Mues, E. $\quad$ Uber ein Problem von Hayman, Math. Z., 164, 239-259 (1979).

[16] Nevanlinna, R. Le théorème de Picard-Borel et la théorie des fonctions méromorphes. Gauthiers-Villars, Paris (1929).

[17] Ojeda, J. On Hayman's Conjecture over a p-adic field, Taiwanese Journal of Mathematics 12 (9), p. 2295-2313, (2008).

[18] Ojeda, J. Applications of the p-adic Nevanlinna theory to problems of uniqueness, Advances in p-adic and Non-Archimedean analysis. Contemporary Mathematics 508, p.161-179 (2010). 